\title{
A rare polymorphism in the low density lipoprotein (LDL) gene that affects mRNA splicing
}

\author{
M. Bourbon ${ }^{\mathrm{b}}$, X-M. Sun ${ }^{\mathrm{a}}$, A.K. Soutar ${ }^{\mathrm{a}, *}$ \\ ${ }^{a}$ MRC Clinical Sciences Centre, Imperial College London, London, United Kingdom \\ ${ }^{\mathrm{b}}$ Instituto Nacional de Saúde Dr. Ricardo Jorge, Lisbon, United Kingdom
}

Received 12 December 2006; received in revised form 17 January 2007; accepted 19 January 2007

Available online 28 February 2007

\begin{abstract}
Familial hypercholesterolaemia $(\mathrm{FH})$ is usually caused by mutations in the low density lipoprotein (LDL) receptor gene (LDLR) that impair clearance of LDL from the circulation. The increased risk of premature coronary heart disease associated with FH can be reduced by dietary advice and treatment with lipid-lowering drug therapy, but it is important to identify affected individuals at an early stage. Several programmes for genetic diagnosis of FH that rely on identifying nucleotide substitutions in genomic DNA have been initiated, but the validity of these is dependent on distinguishing between a silent nucleotide variant and a mutation that affects LDL-receptor function. Here we describe a single nucleotide substitution in the coding region of exon 9 of $L D L R$ that is an apparently silent polymorphism: CGG (Arg406) to AGG (Arg). Analysis of mRNA from the patient's cells showed that the mutation introduces a new splice site that is used to the exclusion of the natural splice site and causes a deletion of $31 \mathrm{bp}$ from the mRNA, predicted to introduce premature termination four codons after R406. This finding emphasizes the caution needed in genetic diagnosis of FH based on genomic DNA sequence alone.
\end{abstract}

(C) 2007 Elsevier Ireland Ltd. All rights reserved.

Keywords: Familial hypercholesterolaemia; Genetic screening; DNA-based diagnosis

\section{Introduction}

Familial hypercholesterolaemia (FH) is caused by defective clearance of low density lipoproteins (LDL) from the circulation, leading to increased levels of serum LDL and markedly increased risk of atherosclerosis and premature coronary heart disease [1]. FH is classically caused by dominant loss-of-function mutations in the gene for the LDL receptor, and occurs with a frequency of about 1/400-500 in most populations, making heterozygous $\mathrm{FH}$ one of the commonest inherited single gene disorders. Homozygous FH is very rare, and presents with a much more severe phenotype [1]. One widespread and two very rare heterozygous mutations in the gene for apolipoprotein $\mathrm{B}$, the major protein

\footnotetext{
* Corresponding author at: Lipoprotein Group, MRC Clinical Sciences Centre, Imperial College London, Hammersmith Hospital, Du Cane Road, London W12 0NN, United Kingdom. Tel.: +44 208383 2324; fax: +442083832028.

E-mail address: anne.soutar@csc.mrc.ac.uk (A.K. Soutar).
}

of LDL that is the ligand recognized by the LDL receptor, cause essentially the same phenotype as heterozygous $\mathrm{FH}$, although the disorder is generally referred to as familial defective apolipoprotein B (FDB) [2].

More recently, defects in other genes have been identified that impair the normal function of the LDL receptor pathway, for example recessive loss-of-function mutations in ARH [3] and dominant gain-of-function mutations in PCSK9 [4], but these remain relatively rare causes of inherited hypercholesterolaemia. In contrast, loss-of-function mutations in PCSK9 are associated with a $40 \%$ reduction in serum cholesterol and highly significant protection from coronary heart disease [5]. This finding has emphasized that avoiding coronary heart disease depends on maintaining a low plasma cholesterol level from early in life [6].

The risk of coronary disease in FH can be significantly reduced by cholesterol-lowering therapy with statins, and the availability of these drugs has markedly improved prognosis for FH patient [7]. However, it is important to commence preventative advice and treatment at a young age and this can be 
aided by identification of the underlying genetic defect, which allows unambiguous identification of affected relatives. Indeed, the relative ease with which variants in genes can now be detected by high throughput sequencing of amplified genomic DNA has allowed identification of numerous different mutations in the LDL-receptor gene in FH patients [8-10]. Clearly if this information is to be used for diagnostic purposes it is of the utmost importance that the effect of a potential mutation on LDL receptor function is known [11], but this has not always been ascertained and some "mutations" have been reported that do not cause hypercholesterolaemia. Equally well, the effect of rare apparently silent mutations should be investigated. We demonstrate this here by reporting a mutation in exon 9 of the LDL-receptor gene that is not predicted to cause an amino acid substitution, but does introduce a new splice site. This results in deletion of $31 \mathrm{bp}$ from exon 9, predicted to introduce a premature termination codon and to result in a truncated protein with no residual function.

\section{Materials and methods}

All the methods for sequencing LDL-receptor mRNA from EBV-transformed lymphocytes and genomic DNA from whole blood have been published previously [12]. PCR products were cloned into T-vector for sequencing with the Sp6 or T7 primers according to the supplier's instructions (Promega).

\section{Results and discussion}

The index patient attended the Hammersmith Hospital Lipid Clinic and had a diagnosis of probable heterozygous FH
[7], based on an untreated plasma cholesterol of $8.7 \mathrm{mmol} / 1$, the presence of tendon xanthomas and angina since the age of 29 years. When the LDL-receptor gene in this patient was amplified from genomic DNA and sequenced, the patient was found to be heterozygous for two common polymorphisms, StuI in exon 8 [13] and MspI in exon 15 [14], but the only novel variant observed was a heterozygous single base substitution in codon R406 in exon 9 (where 1 is the Met initiator codon).

Since this base substitution changed codon 406 (R385 in the mature protein) from CGG to AGG, which is also predicted to encode an Arg residue, it was at first assumed to be a silent polymorphism. However, since no other potential defects in $L D L R$ were found in this patient, the possibility that this variant was pathogenic was explored, and inspection of the sequence revealed the presence of a possible cryptic splice site activated by this single base substitution (Fig. 1). Calculation of the "splice site score" suggested that the new splice site (score 8.0) was at least as likely to be used as the natural splice site (score 6.7)

To determine whether the nucleotide variant in exon 9 did affect splicing, a fragment of LDL receptor mRNA was amplified by RT-PCR from RNA isolated from Epstein-Barr virus (EBV)-transformed lymphocytes from the patient and its nucleotide sequence was determined. As shown in Fig. 2A, the sequence revealed the presence of a heterozygous deletion in exon 9 of the mRNA, although the "mutant" mRNA was only present at a low level. The extent or nature of the deletion in the mRNA was not clear, and thus the amplified mutant fragment was cloned and sequenced. This showed that the mutant mRNA comprised exon 8 spliced to part way along exon 9, with the first 31 bp of exon 9 deleted from the mature mRNA, and that the mutant allele carried the common $\mathrm{G}$ of the StuI polymorphism (Fig. 2B).

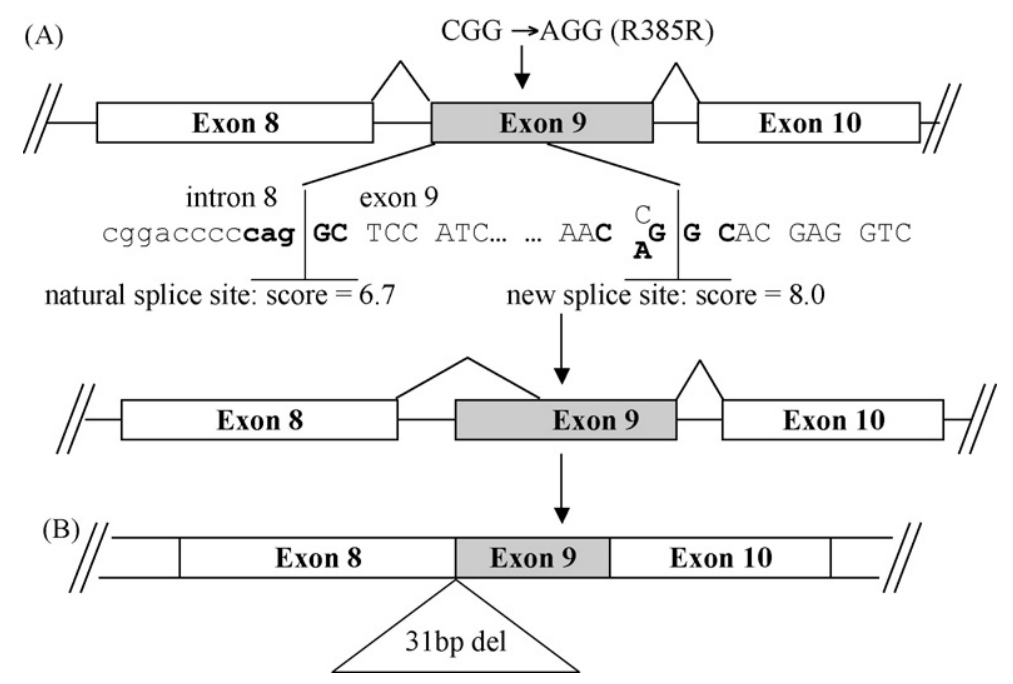

Fig. 1. Potential splice cryptic site in exon 9 of the LDL receptor gene of an FH patient. (A) Diagram of part of the LDL receptor gene with exons represented as boxes and introns by lines, with the nucleotide substitution in codon 406 found in the patient indicated by an arrow above exon 9 . The sequence of the intron:exon junction between exons 8 and 9 is shown, and that surrounding the base substitution in codon 406 . The splice scores for the natural splice site and the new potential splice sites were calculated according to Zhang (http://rulai.cshl.edu/new_alt_exon_db2/HTML/score.html), based on the algorithm of Shapiro and co-workers [18]. (B) Use of the new splice site is predicted to result in deletion of $31 \mathrm{bp}$ from the start of exon 9. 
(A) Sequence of mRNA

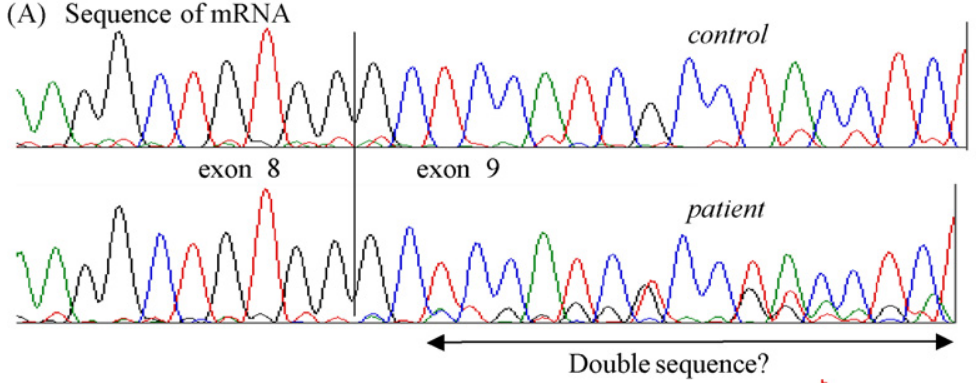

(B) Sequence of cloned RT-PCR fragments control

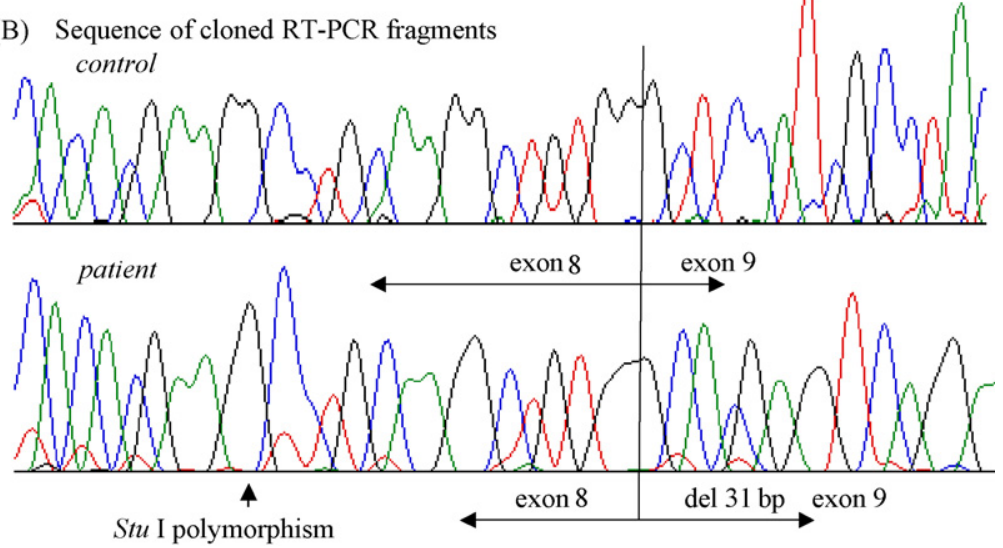

Fig. 2. Nucleotide sequence of amplified LDL receptor mRNA. (A) Total mRNA was isolated from EBV-transformed lymphocytes from the patient and from a control subject, and a fragment encompassing exons 7-18 (approximately $1.7 \mathrm{~kb}$ ) was amplified by RT-PCR and sequenced with a primer in exon 7. The junction between exons 8 and 9 is indicated, together with the region in the patient where there appears to be double sequence, indicative of a heterozygous deletion in the mRNA. (B) The amplified fragments were cloned and sequenced; the sequences shown are from the normal length fragment from the control subject and the shorter fragment of the patient's mRNA; the site of the 31 bp deletion of exon 9 is indicated.

To determine whether the splicing defect was partial, i.e. whether only part of the mutant mRNA was mis-spliced, we compared the sequences surrounding two polymorphic sites in the patient's genomic DNA and transcribed mRNA. As can be seen from Fig. 3, this showed that the majority of the mRNA was derived from one allele only; in the case of the StuI polymorphism in exon 8, this is known to be the "normal" allele (Fig. 2B), suggesting that little or no full length mRNA from the mutant allele is present in the total mRNA (Fig. 3A). If splicing were only partially defective, the proportion of the two bases at the polymorphic sites would be equivalent in total
mRNA. Similarly, at the MspI polymorphic site, there was a much lower than expected amount of the $\mathrm{G}$ allele, presumably present on the mutant allele of the $L D L R$ (Fig. 3B)

Thus we conclude that the single base substitution in exon 9 introduces a new splice site that is used to the exclusion of the normal splice site; this is important because some cryptic splice sites can be used to only a small extent. The reduced amount of the aberrantly spliced mRNA is probably due to nonsense-mediated decay [15], because the 31 bp deletion is predicted to introduce a premature termination codon into the mRNA five codons after G396, the end of exon 8.

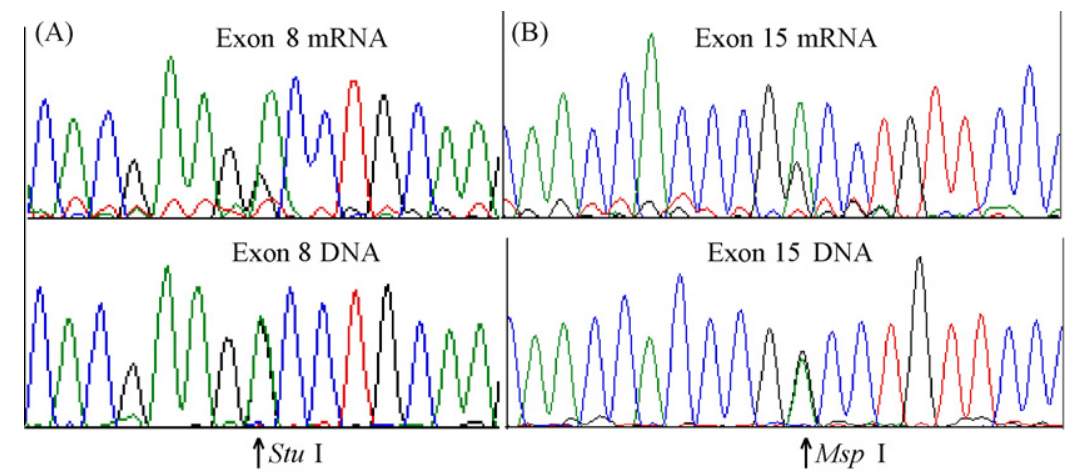

Fig. 3. Nucleotide sequences of amplified genomic DNA and mRNA from the patient. A. Sequence of a fragment of exon 8, showing the StuI polymorphism [13]. (B) Fragment of exon 15, showing the MspI polymorphism [14]. 
Reviewing previous LDL-receptor gene sequencing data, we noted that the same heterozygous base substitution was present in the genomic DNA of a Chinese homozygous FH patient in whom we believed we had not detected any defect in the LDL-receptor gene (subject no. 1 described by Sun et al. [16]). No cells were available to us from this Chinese patient, and at that time we had failed to note that the nucleotide substitution might introduce a splice site and had dismissed it as a rare silent polymorphism. The difference in origins of the two patients suggests that this mutation is very unlikely to have been inherited from a common ancestor, and since it has recurred, it may be in other populations as well [17]. Our findings again emphasize the care that must be taken before reporting the presence or absence of a mutation in the LDL-receptor gene for diagnostic purposes.

\section{References}

[1] Goldstein JL, Hobbs HH, Brown MS. The metabolic and molecular bases of inherited disease. New York, NY: McGraw Hill; 2001. pp. 2863-2913.

[2] Myant NB. Familial defective apolipoprotein B-100: a review, including some comparisons with familial hypercholesterolaemia. Atherosclerosis 1993;104:1-18.

[3] Soutar AK, Naoumova RP, Traub LM. Genetics, clinical phenotype, and molecular cell biology of autosomal recessive hypercholesterolemia, Arterioscler. Thromb Vasc Biol 2003;23:1963-70.

[4] Sun XM, Eden ER, Tosi I, et al. Evidence for effect of mutant PCSK9 on apolipoprotein B secretion as the cause of unusually severe dominant hypercholesterolaemia. Hum Mol Genet 2005;14:1161-9.

[5] Cohen J, Pertsemlidis A, Kotowski IK, Graham R, Garcia CK, Hobbs HH. Low LDL cholesterol in individuals of African descent resulting from frequent nonsense mutations in PCSK9. Nat Genet 2005;37:161-5.

[6] Cohen JC, Boerwinkle E, Mosley Jr TH, Hobbs HH. Sequence variations in PCSK9, low LDL, and protection against coronary heart disease. N Engl J Med 2006;354:1264-72.
[7] Scientific Steering Committee on behalf of the Simon Broome Registry. Mortality in treated heterozygous familial hypercholesterolaemia: implications for clinical management. Atherosclerosis 1999; 142:105-12.

[8] Heath KE, Gahan M, Whittall RA, Humphries SE. Low-density lipoprotein receptor gene (LDLR) world-wide website in familial hypercholesterolaemia: update, new features and mutation analysis. Atherosclerosis 2001;154:243-6.

[9] Stenson PD, Ball EV, Mort M, et al. Human gene mutation database (HGMD): 2003. Hum Mutat 2003;21:577-81, update.

[10] Villeger L, Abifadel M, Allard D, et al. The UMD-LDLR database: additions to the software and 490 new entries to the database. Hum Mutat 2002;20:81-7.

[11] Naoumova RP, Neuwirth C, Pottinger B, Whittal R, Humphries SE, Soutar AK. Genetic diagnosis of familial hypercholesterolaemia: a mutation and a rare non-pathogenic amino acid variant in the same family. Atherosclerosis 2004;174:67-71.

[12] Sun XM, Patel DD, Knight BL, Soutar AK. Comparison of the genetic defect with LDL-receptor activity in cultured cells from patients with a clinical diagnosis of heterozygous familial hypercholesterolemia. The Familial Hypercholesterolaemia Regression Study Group. Arterioscler Thromb Vasc Biol 1997;17:3092-101.

[13] Kotze MJ, Retief AE, Brink PA, Weich HFH. A DNA polymorphism in the human low-density lipoprotein receptor gene. South Afr Med J 1986;70:77-9.

[14] Giesel J, Weisshaar B, Oette K, Mechtel M, Doerfler W. Double MspI RFLP in the human LDL receptor gene. Nucleic Acids Res 1987;15:3943.

[15] Hentze MW, Kulozik AE. A perfect message: RNA surveillance and nonsense-mediated decay. Cell 1999;96:307-10.

[16] Sun XM, Patel DD, Webb JC, et al. Familial hypercholesterolemia in China. Identification of mutations in the LDL-receptor gene that result in a receptor-negative phenotype. Arterioscler Thromb 1994;14:8594.

[17] Bourbon M, Fowler AM, Sun XM, Soutar AK. Inheritance of two different alleles of the low-density lipoprotein (LDL)-receptor gene carrying the recurrent Pro664Leu mutation in a patient with homozygous familial hypercholesterolaemia. Clin Genet 1999;56:225-31.

[18] Senapathy P, Shapiro MB, Harris NL. Splice junctions, branch point sites, and exons: sequence statistics, identification, and applications to genome project. Methods Enzymol 1990;183:252-78. 